\title{
Clinical pharmacology consultations for safety, education and profit
}

\section{Loretta S Loftus, ${ }^{1}$ Gerald H Sokol ${ }^{2}$}

Clinical pharmacologists enjoy an active professional life in the academic, government and pharmaceutical industry arenas but they also belong in the

'Medicine and Oncology, University of South Florida College of Medicine and $\mathrm{H}$ Lee Moffitt Cancer Center, Tampa, Florida, USA

${ }^{2}$ Medicine and Clinical Pharmacology, Uniformed Services University, Bethesda, Maryland, USA

Correspondence to Dr LS Loftus, Professor, Medicine and Oncology, University of South Florida College of Medicine and H Lee Moffitt Cancer Center, 12902 Magnolia Drive, Tampa, Florida 33612, USA; loretta.loftus@moffitt.org

\section{UNLOCKED}

This paper is freely available online under the BMJ Journals unlocked scheme, see http:// ejhp.bmj.com/info/unlocked.dtl community to provide consultations for the complexities of patient care in hospitals and professional clinics. The Institute of Medicine's (IOM) landmark report, To err is human: building a safer health system, brought the significance of medical errors and need for healthcare safety to the forefront. As many as 44 000-98 000 people each year were estimated to die in hospitals as a result of medical errors at that time. Medical error, defined as the failure of a planned action to be completed as intended or the use of a wrong plan to achieve an aim, included adverse drug events, improper transfusions, surgical injuries, wrong site surgery, suicides, restraint related injuries or death, falls, burns, pressure ulcers and mistaken patient identities. ${ }^{1}$ Medication errors pose a major threat to patient safety and occurred with significant frequency in $5.22 \%$ of patients admitted annually to hospitals in one study. ${ }^{2}$ The cost in human lives from preventable medical errors is staggering enough but there is the additional financial cost due to prolonged hospitalisations, lost household income, productivity and disability, estimated at between $\$ 17$ billion and $\$ 29$ billion annually by the IOM. Additionally, there is cost in terms of loss of trust in the healthcare system. Research and education to assure the safe use of medications were the tools promoted to reduce medical errors in the IOM report. ${ }^{1}$

The reasons for medication errors are varied and stem from mistakes in prescribing, dispensing, labelling and administration. ${ }^{3}$ Recent medical school graduates may often be involved in errors of prescription. ${ }^{4}$ Recent changes in medical school curricula where emphasis has been 
placed on instruction in the social sciences and reduction in factual information may have created an adverse effect on clinical pharmacology exposure, a discipline that is more factually based and not organ based. ${ }^{5}$

A study by Bond et a ${ }^{2}$ identified factors associated with decreased medication errors that included the presence of a drug information service, pharmacist provided adverse drug reaction management and drug protocol management, pharmacist participation on medical rounds, pharmacist provided admission histories and increased staffing of clinical pharmacists/occupied bed. As staffing increased for clinical pharmacists/ occupied hospital bed, medication errors decreased by $286 \%{ }^{2}$

Clinical pharmacologists and clinical pharmacists share common ground in the principles of drug therapy and goals to ensure the safety and efficacy of treatments for patients. Both professional groups are accountable for patients and compete for the same financial resources. However, there are clear differences, especially in undergraduate and postgraduate training. Most clinical pharmacologists are physicians with postgraduate training in a medical specialty and fellowship in clinical pharmacology. Clinical pharmacists have undergraduate degrees in pharmacy with a shorter duration of postgraduate training in clinical pharmacy. Although clinical pharmacology has developed into a specialty of medicine for over 40 years, its impact on healthcare services has been less evident. Clinical pharmacy services have been more visible but have been less notable as an academic discipline. Research in drug development, medical education, execution of clinical trials and government regulatory affairs for drug approval have been the realm of the clinical pharmacologists whereas clinical pharmacists predominantly function as a supportive service in hospital rounds and clinics. Domination of one group over the other may depend on the country worldwide. Collaboration between clinical pharmacologists and clinical pharmacists is necessary to benefit patient care and safety, education and research. ${ }^{6}$

A previous impediment to the delivery of collaborative drug therapy management services by clinical pharmacists and physicians was concern about the cost and reimbursement for these services. Reimbursement for clinical pharmacology consultations varies among countries. For instance, compensation to providers of medical care in the UK is predominantly (85.5\%) funded through the government regulated National Health Service. Funds are derived through taxation. Healthcare providers are government employees who are paid salaries or a fee set from a contract. On the other hand, reimbursement for medical services in the USA may come from multiple sources, most often through the purchase of private insurance by employees and employers. Public funding from taxation for government operated Medicare and Medicaid programmes accounts for approximately $44.7 \%$. Healthcare providers are reimbursed for the individual service provided. ${ }^{7}$ Medication therapy management codes in the USA are available from the Centres for Medicare and Medicaid Services and the American Medical Association. ${ }^{89}$ These codes relate to initial and subsequent encounters with patients for medication therapy management services provided by pharmacists and physicians. Reimbursement depends on proper documentation in the medical record of the services provided. The charge corresponds to the level of complexity of the service provided, including counselling and time spent with the patient, as in any medical care event. Billing for the collaborative drug therapy management services requires the use of the physician's unique provider identification number, the current procedural terminology codes for evaluation and management, other standard codes for healthcare setting and other demographic information. ${ }^{10}$

Patients often do not understand why they take certain medications, the side effects associated with these pharmacological agents and the potential drug-drug interactions. Furthermore, polypharmacy is rampant, and patients are often unaware of drug administration schedules, especially when they are infirm and/or cognitively impaired. Drug information distributed at pharmacies is verbose and unintelligible to many patients. Few US medical schools offer extensive pharmacology training at a time when therapeutics are more complicated than ever and will likely escalate. Clinical pharmacists must work in tandem with clinical pharmacologists, and these clinical pharmacology services need to be available to outpatient clinics, private practices and hospital based medical practices as well as to hospital inpatient venues. Clinical pharmacists with their profound knowledge of drugs and ready access to drug databases complement the clinical pharmacologists who provide their union of drug knowledge, physical diagnosis and clinical skills. The development and expanded use of clinical pharmacology consultation services in a variety of settings will create greater safety for patients, provide educational benefits to physician colleagues, other health professionals and patients, and may even become a profit centre for medical care.

\section{Competing interests None.}

Provenance and peer review Commissioned; externally peer reviewed.

European Journal of Hospital Pharmacy

2012;19:21-22.

doi:10.1136/ejhpharm-2011-000004

\section{References}

1. Kohn KT, Corrigan JM, Donaldson MS. To err is human: building a safer health system. Washington, DC: National Academy Press 2009:1-16.

2. Bond CA, Raehl CL, Franke T. Clinical pharmacy services, hospital pharmacy staffing and medication errors in United States hospitals. Pharmacotherapy 2002;22:134-47.

3. Williams DJP. Medication errors. J R Coll Physicians Edinb 2007;37:343-6.

4. Ross S, Bond C, Rothnie $\mathrm{H}$, et al. What is the scale of prescribing errors committed by junior doctors? A systematic review. Br J Clin Pharmacol 2009;67:629-40.

5. Likic R, Maxwell SR. Prevention of medication errors: teaching and training. Br J Clin Pharmacol 2009;67:656-61.

6. Golocorbin-Kon S, Lalic M, Raskovic A, et al. Clinical pharmacology and clinical pharmacy: competition or collaboration? Ther Pharmacol Clin Toxicol 2009;13:33-40.

7. Roe AM, Liberman A. A comparative analysis of the United Kingdom and the United States health care systems. Health Care Manag (Frederick) 2007;26:190-212.

8. Centers for Medicare and Medicaid Services. https:// www.cms.gov/ (accessed 20 December 2011).

9. American Medical Association. http://www. ama-assn.org (accessed 20 December 2011).

10. Kuo GM, Buckley TE, Fitzsimmons DS, et al. Collaborative drug therapy management services and reimbursement in a family medicine clinic. Am J Health Syst Pharm 2004;61:343-54. 